\title{
Subjective well-being, drug attitude, and changes in symptomatology in chronic schizophrenia patients starting treatment with new-generation antipsychotic medication
}

\author{
Christian G. Widschwendter", Georg Kemmler, Maria A. Rettenbacher, Nursen Yalcin-Siedentopf and Alex Hofer
}

\begin{abstract}
Background: Non-adherence to medication remains a major challenge in the long-term management of patients with schizophrenia. Next to lack of insight into the illness, adverse effects of antipsychotic drugs, cognitive deficits, poor therapeutic alliance, reduced quality of life, missing social support, and negative attitudes toward medication are predictors of non-adherence. This study examined potential correlations between attitudes toward antipsychotic drug therapy, subjective well-being, and symptom change in patients with chronic schizophrenia.

Methods: 30 patients with schizophrenia starting monotherapy with a new-generation antipsychotic were included into the study. The Drug Attitude Inventory (DAI) and the Subjective Well-being under Neuroleptic Treatment Scale, short form (SWN-K), were administered after 2, 4, and 12 weeks of treatment. At the same points in time and at baseline, psychopathological symptoms were rated by means of the Positive and Negative Syndrome Scale (PANSS), and functioning was assessed by means of the Global Assessment of Functioning Scale (GAF). Antipsychotic induced side effects were evaluated by using the Udvalg for Kliniske Undersogelser (UKU) Side Effect Rating Scale.

Results: Study participants had a mean age of $37.5 \pm 9.7$ years, baseline symptoms were mild. The PANSS total score improved significantly from baseline to weeks $4(p=.003)$ and $12(p=.001)$, respectively. Neither the DAl total score nor the SWN-K total score changed significantly over the course of time. The severity of symptoms was not correlated with drug attitude at any time point but was negatively correlated with wellbeing at weeks $2(r=-.419, p=.021)$ and 4 $(r=-.441, p=.015)$. There was no significant correlation between DAl and SWN-K total scores at any time point.

Conclusions: Next to showing that the DAI and the SWN-K measure different aspects of subjective experiences during antipsychotic treatment these findings emphasize the use of both instruments to optimize adherence to medication.
\end{abstract}

\section{Background}

Despite advances in the pharmacotherapy of schizophrenia poor-adherence to antipsychotic medication remains a major challenge in the long-term treatment of patients. Most studies reported on high frequencies of partial and non-adherence in this patient group with different factors being relevant in this context [1]: next to lack of insight

\footnotetext{
* Correspondence: christian.widschwendter@i-med.ac.at

Medical University Innsbruck, Department of Psychiatry,Psychotherapy, and

Psychosomatics. Division of Psychiatry I, Anichstrasse 35, 6020 Innsbruck, Austria
}

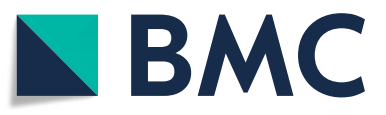

(c) The Author(s). 2018 Open Access This article is distributed under the terms of the Creative Commons Attribution 4.0 International License (http://creativecommons.org/licenses/by/4.0/), which permits unrestricted use, distribution, and reproduction in any medium, provided you give appropriate credit to the original author(s) and the source, provide a link to the Creative Commons license, and indicate if changes were made. The Creative Commons Public Domain Dedication waiver (http://creativecommons.org/publicdomain/zero/1.0/) applies to the data made available in this article, unless otherwise stated.

into the illness negative attitudes towards the illness and the medication, negative past experiences with the illness and its treatment, and the lack of support systems are known to be strong predictors of non-adherence [2-5]. This is of particular relevance, since non-adherence is one of the most important risk factors for relapse, rehospitalization, and treatment resistance as well as for substance abuse, violence, arrests, suicide attempts, and impaired long-term functioning [6]. During the era of first generation antipsychotics, the emphasis of treatment lay 
on objective outcome parameters, e.g., a reduction of positive symptoms, and little research was done with regard to the patients' subjective perspective [7]. With the introduction of new generation antipsychotics treatment goals became more ambitious and more attention was paid to patients' complaints, e.g., cognitive slowing, affective blunting or loss of spontaneity and volition $[8,9]$. Next to that, the interest in the patients' view of quality of life has significantly increased over the last decades [10].

Most patients with schizophrenia, if not acutely psychotic or experiencing severe cognitive impairments, are able to complete self-rating scales in a meaningful way, and it has been shown that patients' and psychiatrists' perspectives on antipsychotic treatment differ considerably [1113]. Several scales have been developed to assess quality of life in patients with schizophrenia [7] and both the impact of pharmacological therapy on quality of life and the relationship between subjective experience and attitudes and adherence to medication attracted attention over the past decades. In 1983, Hogan et al. developed the Drug Attitude Inventory (DAI) with the objective to quantify patient's subjective experience of treatment with antipsychotic drugs [14]. Importantly, positive attitudes towards medication have been associated with lower symptom levels, and treatment response has been demonstrated to be positively correlated with a positive drug attitude [3, 15-19]. Later, Naber et al. developed the "Subjective Well-being under Neuroleptic Treatment" scale (SWN), which captures the subjective experiences of patients during antipsychotic drug treatment $[12,20]$ and has inconsistently been shown to correlate with current symptomatology or changes in psychopathology [20-22].

Although some authors claimed that both instruments examine quality of life $[7,23]$, merely the SWN is highly correlated with this subjective outcome variable [12]. Initially designed to predict drug compliance, the DAI focuses on the subjective effects of antipsychotic medication and on values and attitudes toward the illness and health without differentiating between these issues [24]. The SWN, on the other hand, measures quality of life rather than experiences attributed to antipsychotic medication without distinguishing between pharmacogenic or morbogenic components [7]. However, consensus on a uniform definition of the multidimensional and partially subjective concept of quality of life [25-27] is still missing. In contrast to "overall quality of life", the term "health-related quality of life" is used in the context of medical treatment and research and includes three major determinants: subjective well-being, functioning in daily life, and external resources. Consequently and in contrast to the concept of "well-being", which can be seen as a psychological and emotional state, the concept of quality of life also encompasses physical, social, cognitive and functional aspects [24, 28].
The aim of this study was to assess the associations between subjective well-being and attitudes towards antipsychotic medication as well as the interrelations between these issues and both symptoms and functioning in patients with chronic schizophrenia starting monotherapy with a new-generation antipsychotic drug.

\section{Methods}

\section{Subjects and experimental design}

Patients aged between 18 and 65 years and starting treatment with a new-generation antipsychotic drug were included into a prospective longitudinal study to build a drug monitoring register. Subjects were recruited via a specialized outpatient service for patients with psychotic disorders or they were inpatients. They met the diagnostic criteria of schizophrenia, schizotypal and delusional disorders according to ICD-10 and signed informed consent forms as approved by the local ethics committee. They did not suffer from any other axis I disorder, including substance abuse. Diagnoses were confirmed using chart information and reports from clinicians who had treated these patients. Patients who had previously been receiving antipsychotic medication underwent a washout period of 3-5 days. Antipsychotics were chosen by the psychiatrists treating the patients, dosing followed clinical needs within the recommended labeled dose ranges.

At baseline as well as after 2, 4, and 12 weeks of treatment, psychopathology was rated by means of the Positive and Negative Syndrome Scale (PANSS) [29]. At the same points in time, the severity of illness and overall level of functioning were assessed using the Global Assessment of Functioning Scale (GAF) [30, 31]. Additionally, side effects were quantified using the Udvalg for Kliniske Undersogelser (UKU) Side Effect Rating Scale [32] at weeks 2, 4, and 12. The UKU comprises a total of 48 symptoms, arranged into four groups: psychic, neurological, autonomic and other side effects. Each symptom is scored on a severity scale from 0 to 3 , and the rater assesses whether the report is best attributed to a side effect (rated as improbable, possible or probable) or related to the disease. Only adverse effects with scores $\geq 1$ on any UKU item and a causal relationship of possible or probable, were considered as antipsychotic induced side effects.

After 2, 4, and 12 weeks of treatment, the patients' subjective well-being as well as their subjective response to and their attitudes towards medication were assessed by means of the short form of the SWN (SWN-K) and the DAI, respectively.

The SWN-K represents a self-rating Likert scale with 6 response categories $(1=$ not at all, $6=$ very much) [20]. It consists of 20 statements (10 positive and 10 negative) on 5 subscales ("mental functioning", "emotional regulation", "social integration", "physical functioning", "self-control") 
with a minimum total score of 20 and a maximum total score of 120; higher scores indicate better well-being. In accordance with the guidelines, scoring of negatively worded items was reversed $(1 \rightarrow 6,2 \rightarrow 5, \ldots, 6 \rightarrow 1)$ before total score and subscores were calculated [20]. The criterion for adequate subjective well-being is met if an SWN-K total score $\geq 80$ is achieved [33, 34].

The DAI, on the other hand, is a 30-item self-report questionnaire consisting of statements about perceived effects and benefits of antipsychotics with which the patient can agree or disagree. It is divided into 7 factors: (1) subjective positive feelings related to antipsychotics (e.g., feeling happier), (2) subjective negative feelings attributed to the drugs (e.g., feeling tired and sluggish), (3) health/illness-dependent drug intake: patients' model of health (e.g., believing it is unnatural to take medication), (4) patients' confidence in the physician (e.g., believing it is up to the doctor when one stops taking medication), (5) control: patients' attitudes toward the locus of control in taking medication (e.g, feeling pressured to ingest medication), (6) prevention: patients' belief in the effect of antipsychotics in forestalling relapse (e.g., antipsychotics can prevent getting sick), and (7) harm: patients' concerns with potential toxic effects (e.g., believing medication is a slow acting poison). Each item oft the DAI is scored 1 or 2 , depending on whether the answer selected by the patient indicates a negative or positive view of medication. Raw scores of the DAI (total score and subscales) were linearly transformed to a common range from 0 to 100 to facilitate interpretation [35]. For the total score and for those DAI subscales addressing positive aspects, higher scores reflect a more positive drug attitude. For DAI subscales dealing with negative aspects higher scores indicate a more negative drug attitude.

\section{Statistical methods and power analysis}

Prior to the analysis all metric variables were checked for deviations from normality by investigating their skewness, $G_{1}$. Values of $G_{1}$ above 0.5 or below -0.5 were regarded as sizable deviation from normality indicating the need for non-parametric testing. Changes of PANSS, GAF, SWN-K and DAI over the course of time were analyzed by repeated-measures ANOVA or by Friedman test and subsequent Wilcoxon matched-pairs tests, depending on the variable type (normally or non-normally distributed, respectively). Missing observations were omitted listwise in the repeated-measures analyses (i.e., if one time point was missing the patient as a whole was disregarded in the respective analysis). However, missing values were very rare as all patients completed the SWN-K and 29 of 30 (96.7\%) completed the DAI at all planned assessment times (week 2, 4 and 12). Associations of SWN-K and DAI scores with patient characteristics, symptomatology (PANSS), side effects (UKU) and GAF were investigated by means of the Spearman rank correlation coefficient, as part of the variables involved were non-normally distributed.

The sample size of $n=30$ patients provides sufficient power $(1-\beta=0.8)$ to detect in a repeated-measures ANOVA under standard assumptions (type-one error $\alpha=0.05$; correlations among repeated measures, $r=0.5$; non-sphericity correction, $\varepsilon=0.9$ ) effect sizes of $\mathrm{f}=0.236$ for the comparison of 4 assessments and $\mathrm{f}=0.258$ for comparing 3 assessments. These are medium effect sizes according to Cohen's classification [36]. Moreover, the sample size of $n=30$ is sufficient to detect with a power of $1-\beta=0.8$ and a two-tailed type-one-error of 0.05 , Spearman rank correlation coefficients $r \geq 0.49$ a in a non-parametric correlation analysis. This is a medium to large correlation according to Cohen's classification (close to the threshold of 0.5 indicating large correlations).

\section{Results}

Demographic and clinical characteristics of the study sample are summarized in Table 1 . Data of 30 patients were available for analysis at baseline, week 2 and 4 . At week 12, the DAI evaluation was missing for one subject, and the GAF evaluation for another one. None of the patients withdrew within the 12 weeks observation period. They had a mean age of $37.5 \pm 9.7$ years and a mean duration of illness of $8.7 \pm 6.7$ years with a range from 2 to 21 years. 57\% were male. At baseline, the mean PANSS total score was $59.7 \pm 16.5$, indicating mild symptom severity. The mean baseline GAF score was $56.7 \pm 16.4$, thereby indicating a moderate impairment. At baseline, $40 \%$ of study participants were inpatients, while the others were treated at a specialized outpatient clinic. All patients started monotherapy with a new-generation antipsychotic drug (amisulpride: $n=7$, aripiprazole: $\mathrm{n}=7$, clozapine: $n=6$, olanzapine: $n=3$, quetiapine: $\mathrm{n}=3$, ziprasidone: $n=2$, sertindole: $\mathrm{n}=2$ ). The mean doses and ranges of antipsychotic medication were as follows: amisulpride $428.6 \pm 138 \mathrm{mg}$ (200$600 \mathrm{mg}$ ), aripiprazole $19.3 \pm 15.5 \mathrm{mg}(15-30 \mathrm{mg})$, clozapine $366.7 \pm 75.3 \mathrm{mg}$ (300-500 mg), olanzapine $16.7 \pm$ $5.8 \mathrm{mg} \quad(10-20 \mathrm{mg})$, quetiapine $700 \pm 173.2 \mathrm{mg}$ (600-900 mg), ziprasidone $140 \pm 28.3 \mathrm{mg}$ (120-160 mg), and sertindole $16 \pm 0 \mathrm{mg}$.

The time course of disease severity as indicated by PANSS scores, subjective well-being (SWN-K total score), and attitudes toward medication (DAI total score) is depicted in Table 2. The PANSS total score improved significantly from baseline to weeks 4 and 12, respectively. There was no significant improvement from baseline to week 2 . Both the PANSS positive and general psychopathology subscores improved from baseline to weeks 2, 4, and 12, whereas the PANSS negative 
Table 1 Demographic and Clinical Patient Characteristics $(n=$ 30)

\begin{tabular}{ll}
\hline Age, mean $\pm \mathrm{SD}$, years & $37.5 \pm 9.7$ \\
\hline Sex, $\mathrm{n}(\%)$, female/male & $13(43.3) / 17(56.7)$ \\
Duration of illness, mean \pm SD, years & $8.7 \pm 6.7$ \\
PANSS score at baseline, mean \pm SD & \\
Total score & $59.7 \pm 16.5$ \\
Positive symptoms & $12.1 \pm 5.1$ \\
Negative symptoms & $17.0 \pm 7.1$ \\
$\quad$ General psychopathology & $30.6 \pm 8.1$ \\
GAF score at baseline, mean \pm SD & $56.7 \pm 16.4$ \\
\hline
\end{tabular}

PANSS = Positive and Negative Syndrome Scale; GAF = Global Assessment of Functioning Scale

subscore remained virtually unchanged. The GAF score improved from baseline to weeks 4 and 12. At any assessment, SWN-K and DAI scores reflected both adequate subjective well-being and positive attitudes toward antipsychotic treatment, respectively. Neither SWN-K nor DAI total scores changed significantly over the course of time.

Correlations between SWN-K and DAI and the interrelations between these scales and both symptom level (PANSS) and functioning (GAF) are depicted in Table 3. There was no correlation between SWN-K and DAI total scores at any time point. A significant negative correlation was found between the SWN-K total score and both the total score and the general psychopathology subscore of the PANSS at weeks 2 and 4, but not at week 12. Moreover, the SWN-K total score correlated negatively with the PANSS positive subscore at week 4, whereas no significant correlation was found between the SWN-K total score and the PANSS negative subscore. The DAI total score did neither correlate with the PANSS total score or any of its subscales nor with the GAF score at any point in time. In contrast, the SWN-K total score correlated positively with the GAF score at all time points.

Regarding correlations of patient characteristics, i.e. age, gender, duration of illness, and inpatient vs. outpatient, with SWN-K and DAI scores, no significant correlation was found. Side effects that were most frequently reported within the observation period of 12 weeks (found in $\geq 10 \%$ of patients) were as follows: increased salivation (18.3\%), concentration difficulties (15.8\%), asthenia (15.8\%), weight gain (15\%), tension/ inner unrest (15\%), hypokinesia (12.5\%), failing memory (11.7\%), akathisia (10\%), and diminished sexual desire $(10 \%)$. The item tension/ inner unrest was negatively correlated with the SWN-K total score at weeks $2(r=$ $-.362, p=.05)$ and $12(r=-.454, p=.012)$, and with the DAI total score at week $2(r=-.377, p=.04)$. Additionally, the side effect failing memory was negatively correlated with the SWN-K total score at week $12(r=$ $-.367, p=.046)$. No further correlation between side effects and both subjective well-being and drug attitude was found.

\section{Discussion}

The aim of the current study was to assess a potential association between subjective well-being and attitudes toward antipsychotic medication as well as the interrelations between these issues and both symptom severity and functioning in chronic schizophrenia patients starting treatment with a new-generation antipsychotic drug. As expected, both symptoms and global functioning improved over the course of antipsychotic treatment. Most previous studies reported on an improvement of patients' subjective well-being over the course of treatment [37-39]. In our sample, however, the SWN-K total score did not change over time. Next to a limited sample size this seemingly contradictive finding could be a result of patient selection, since we investigated chronically ill patients experiencing mild baseline symptomatology and a generally positive attitude toward medication as indicated by high mean DAI total scores. Of note, four distinct clusters of subjective well-being have been described in individuals with schizophrenia: $16 \%$ of "patients moderate" "stable high", 31\% "stable moderate", $33 \%$ "stable low", and merely $20 \%$ improving "subjective well-being over the course of treatment" [40]. According to this classification, the patients included into the current study may be seen to have had a stable high subjective well-being, since they consistently achieved a mean SWN-K total score of '80. Following Larsen and Gerlach, we hypothesize that the relatively long mean duration of illness of approximately 9 years may have enabled them to accept their illness [41]. In line with previous studies, we did not find consistent correlations between the PANSS or its subscales and the SWN-K total score [23].

The negative correlation between the SWN-K total score and both the PANSS total score and the general psychopathology subscore is in line with the literature $[42,43]$, and can be explained by the fact that they contain items that include symptoms of anxiety and depression, which may be more critical in influencing subjective well-being rating than any other symptom of schizophrenia.

Our finding of a significant correlation of global functioning with patients' subjective well-being corroborates the results of previous studies $[44,45]$. Accordingly, subjective well-being may directly impact upon a patient's social, psychological, and occupational functioning and vice versa.

Similar to the SWN-K total score and in line with a previous report [46], the DAI total score did not change over 


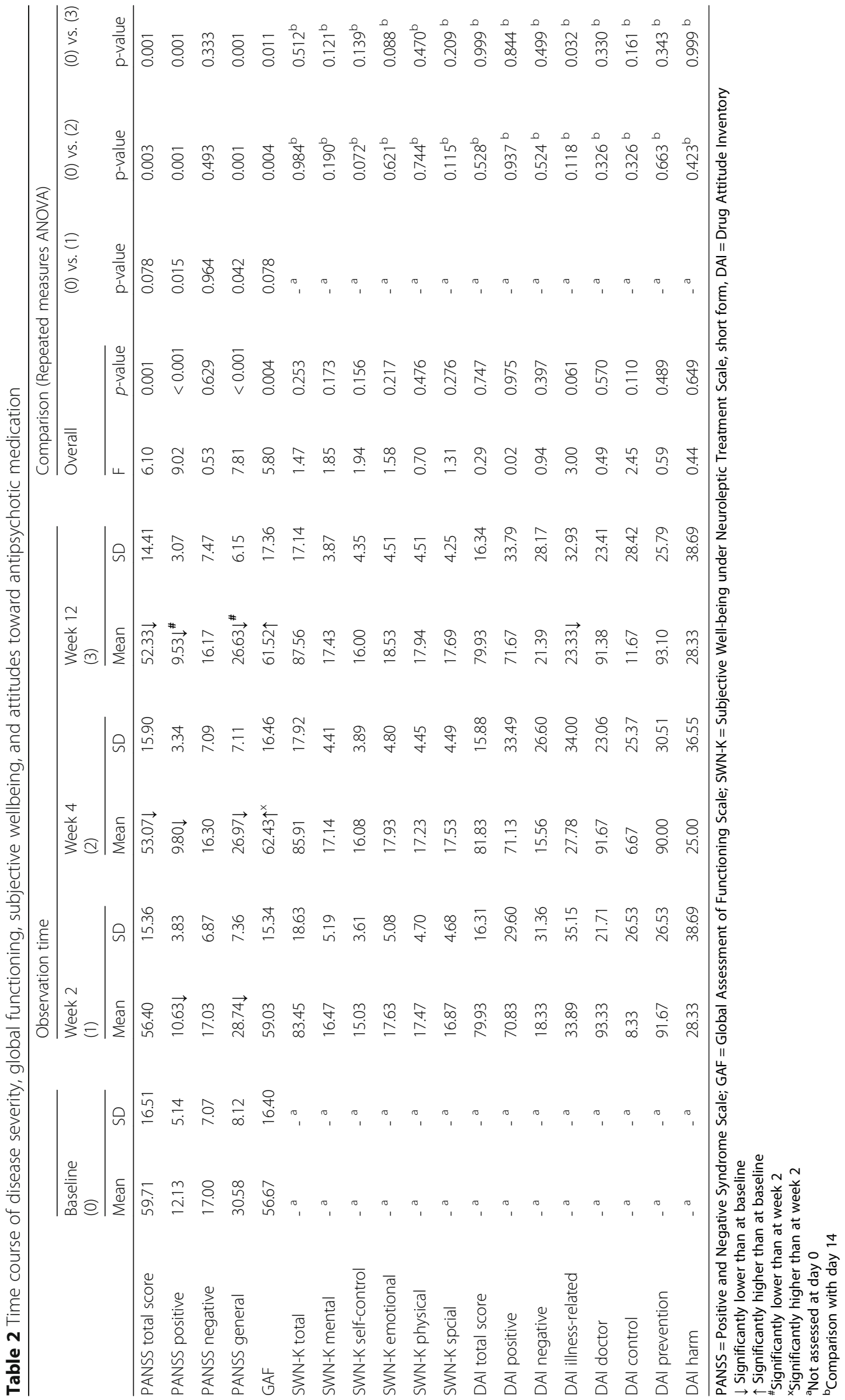




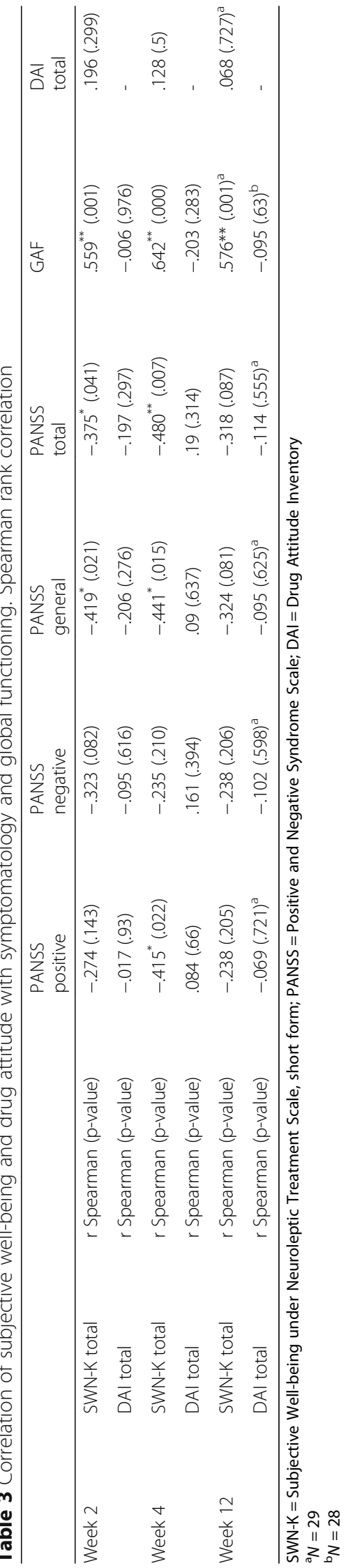


time. Moreover, drug attitude did not correlate with symptom change. Generally, drug adherence has been reported to be predicted by the patients' subjective responses to and attitudes toward antipsychotics [14]. The high mean DAI total score in our sample reflects a general positive attitude toward antipsychotic medication and underscores once more, that we investigated a selected patient sample. It clearly has to be noted that adherence is generally higher among study samples than in routine care and that our findings are therefore not attributable to all individuals suffering from schizophrenia [1].

Notably and in contrast to our finding of a consistent association between patients' subjective well-being and functioning, the DAI total score did not correlate with the GAF score at any time point, which corroborates previous findings [47]. However, in contrast to previous reports we did not find a correlation between the SWN-K and DAI total scores [48, 49]. Next to differences in the patient samples studied, this contradictory result can be explained by the scales' varying scopes. The DAIs' strength lies in measuring compliance and attitudes towards antipsychotic treatment, whereas the SWN-K can be seen as an alternative for a quality of life instrument in patients using antipsychotic medication [48]. One previous study [49] examined a very heterogeneous sample encompassing patients receiving first- and new-generation antipsychotics in different formulations (oral versus long-acting injectable drugs) with $60 \%$ of patients having been on the current medication for at least one year. Furthermore, symptom severity has not been reported in this study. Another longitudinal study [48] investigated patients experiencing more severe symptoms at baseline than our sample. Accordingly, these studies are not entirely comparable with ours.

Regarding the impact of antipsychotic-induced side effects on subjective well-being and drug attitude, inconsistent correlations have been found for tension/ inner unrest and failing memory. This finding is supported by previous research that found that the psychiatrists' beliefs about the tolerance of a particular antipsychotic drug do not necessarily reflect patients' well being and attitude toward medication [50]. $\backslash$ Additionally, this effect can be interpreted as the intense efforts in both inpatient units and specialized outpatient clinic to actively elicit adverse events and to respond to patients' concerns quickly.

When interpreting our data, one has to consider a number of limitations. First of all, the sample size was rather small and early noncompliers, who usually stop attending mental health services, have not been included. Furthermore, the fact that only patients with a generally good attitude toward medication took part in the study limits the generalizability of our findings. Lastly, we did not thoroughly assess the reason for switching antipsychotic medication in patients who had previously been receiving antipsychotic drugs.

\section{Conclusions}

In summary, our findings emphasize that, particularly in chronic schizophrenia patients experiencing mild symptoms, the differences between the SWN and DAI may give good reason for the use of both instruments during antipsychotic treatment in clinical practice and/ or research to systematically monitor and adjust treatment with antipsychotic medication. In so doing it may help finding the optimal dosage and type of antipsychotic medication for an individual patient and therefore enhance adherence.

\section{Abbreviations}

DAl: Drug Attitude Inventory; GAF: Global Assessment of Functioning Scale: PANSS: Positive and Negative Syndrome Scale; SWN: Subjective Well-being under Neuroleptic Treatment Scale; SWN-K: Subjective Well-being under Neuroleptic Treatment Scale, short form

\section{Acknowledgements}

The authors wish to thank the study participants for their contribution to the study.

\section{Availability of data and materials}

The datasets used and/or analysed during the current study are available from the corresponding author on reasonable request.

\section{Authors' contributions}

$\mathrm{AH}$ and CGW initiated, designed and coordinated the study. CW, MAR, NY-S, and $\mathrm{AH}$ conducted data collection, GK helped in analyzing the data, CGW wrote the manuscript, $\mathrm{AH}$ and $\mathrm{G}$ critically revised and approved the final manuscript. All authors read and approved the final manuscript.

\section{Ethics approval and consent to participate}

All procedures contributing to this work complied with the standards of the Ethics Committee of Medical University Innsbruck and were conducted according to Good Clinical Practice standards on human experimentation and with the Helsinki Declaration of 1975, as revised in 2008.

\section{Consent for publication}

No patient identifiable information has been reported.

\section{Competing interests}

Christian Widschwendter, Georg Kemmler, and Nursen Yalcin-Siedentopf have no actual or potential conflict of interest. Maria A. Rettenbacher is member of a advisory board of Eli Lilly and has received speaker's or consultancy fees from Eli Lilly. Alex Hofer has received a research grant from Janssen-Cilag. He has received speaker's or consultancy fees from BristolMyers Squibb, Janssen-Cilag and Lundbeck as well as reimbursements for travel and meeting expenses from Janssen-Cilag and Lundbeck.

\section{Publisher's Note}

Springer Nature remains neutral with regard to jurisdictional claims in published maps and institutional affiliations.

Received: 25 January 2018 Accepted: 12 June 2018 Published online: 28 June 2018

\section{References}

1. Kane JM, Kishimoto T, Correll CU. Non-adherence to medication in patients with psychotic disorders: epidemiology, contributing factors and management strategies. World Psychiatry. 2013;12(3):216-226; doi: https:/doi.org/10.1002/ wps.20060. 
2. Brain C, Allerby K, Sameby B, Quinlan P, Joas E, Karilampi U, Lindström E, Eberhard J, Burns T, Waern M. Drug attitude and other predictors of medication adherence in schizophrenia: 12 months of electronic monitoring $\left(\right.$ MEMS $\left.\left({ }^{\oplus}\right)\right)$ in the Swedish COAST-study. Eur Neuropsychopharmacol. 2013; 23(12):1754-62. 0.1016/j.euroneuro.2013.09.001.

3. Hofer A, Kemmler G, Eder U, Honeder M, Hummer M, Fleischhacker WW Attitudes toward antipsychotics among outpatient clinic attendees with schizophrenia. J Clin Psychiatry. 2002;63(1):49-53.

4. Rettenbacher MA, Hofer A, Eder U, Hummer M, Kemmler G, Weiss EM, Fleischhacker WW. Compliance in schizophrenia: psychopathology, side effects, and patients' attitudes toward the illness and medication. J Clin Psychiatry. 2004;65(9):1211-8

5. Acosta FJ, Hernández JL, Pereira J, Herrera J, Rodríguez CJ. Medication adherence in schizophrenia. World J Psychiatry. 2012;2(5):7482. https://doi.org/10.5498/wjp.v2.i5.74.

6. Hofer A, Fleischhacker WW. Compliance with treatment and its relevance for the management of schizophrenia. Hot Topics Neurol Psychiatry. 2011;11:7-13.

7. Karow A, Naber D. Subjective well-being and quality of life under atypical antipsychotic treatment. Psychopharmacology. 2002;162(1):3-10.

8. Windgassen K. Treatment with neuroleptics: the patient's perspective. Acta Psychiatr Scand. 1992;86(5):405-10.

9. Lewander T. Neuroleptics and the neuroleptic-induced deficit syndrome. Acta Psychiatr Scand Suppl. 1994;380:8-13

10. de Millas W, Lambert M, Naber D. The impact of subjective well-being under neuroleptic treatment on compliance and remission. Dialogues Clin Neurosci. 2006;8(1):131-6.

11. Lambert M, Schimmelmann BG, Karow A, Naber D. Subjective wellbeing and initial dysphoric reaction under antipsychotic drugs concepts, measurement and clinical relevance. Pharmacopsychiatry. 2003;36(Suppl 3):181-90.

12. Naber D. A self-rating to measure subjective effects of neuroleptic drugs, relationships to objective psychopathology, quality of life, compliance and other clinical variables. Int Clin Psychopharmacol. 1995;10(Suppl 3):133-8.

13. Voruganti L, Heslegrave R, Awad AG, Seeman MV. Quality of life measurement in schizophrenia: reconciling the quest for subjectivity with the question of reliability. Psychol Med. 1998;28(1):165-72.

14. Hogan TP, Awad AG, Eastwood R. A self-report scale predictive of drug compliance in schizophrenics: reliability and discriminative validity. Psychol Med. 1983;13(1):177-83.

15. Freudenreich O, Cather C, Evins AE, Henderson DC, Goff DC. Attitudes of schizophrenia outpatients toward psychiatric medications: relationship to clinical variables and insight. J Clin Psychiatry. 2004;65(10):1372-6.

16. Mohamed S, Rosenheck R, McEvoy J, Swartz M, Stroup S, Lieberman JA. Cross-sectional and longitudinal relationships between insight and attitudes toward medication and clinical outcomes in chronic schizophrenia. Schizophr Bull. 2009; https://doi.org/10.1093/schbul/sbn067.

17. Mintz AR, Dobson KS, Romney DM. Insight in schizophrenia: a meta-analysis. Schizophr Res. 1995;18(1):21-8.

18. Kemp RA, Lambert TJ. Insight in schizophrenia and its relationship to psychopathology. Schizophr Res. 2003;61(1):75-88.

19. Hofer A, Rettenbacher MA, Edlinger M, Kemmler G, Widschwendter CG, Fleischhacker WW. Subjective response and attitudes toward antipsychotic drug therapy during the initial treatment period: a prospective follow-up study in patients with schizophrenia. Acta Psychiatr Scand. 2007;116(5):354-61.

20. Naber D, Moritz S, Lambert M, Pajonk FG, Holzbach R, Mass R, Andresen B. Improvement of schizophrenic patients' subjective well-being under atypical antipsychotic drugs. Schizophr Res. 2001;50(1-2):79-88.

21. Putzhammer A, Perfahl M, Pfeiff L, Hajak G. Correlation of subjective well-being in schizophrenic patients with gait parameters, expert-rated motor disturbances, and psychopathological status. Pharmacopsychiatry. 2005;38(3):132-8.

22. Lee Y-W, Kim J-H, Ann J-H, Cho S-E, Lee J, Kim M-J, Cho S-J, Cho I-H, Kim SJ, Lee Y-J, Bae S-M. Subjective well-being in patients with schizophrenia treated with atypical antipsychotics: the impact of psychopathology and adverse drug effects. Clinical psychopharmacology and Neuroscience. 2010; 8(3):149-55

23. Vothknecht S, Schoevers RA, de Haan L. Subjective well-being in schizophrenia as measured with the subjective well-being under neuroleptic treatment scale: a review. Aust N Z J Psychiatry. 2011:45(3): 182-92. https://doi.org/10.3109/00048674.2010.545984.
24. Awad $A G$, Voruganti $L N$. Measuring quality of life in patients with schizophrenia: an update. PharmacoEconomics. 2012;30(3):183-95. https://doi.org/10.2165/11594470-000000000-00000.

25. Spilker B. Quality of life and pharmacogenomics in clinical trials. New York: Lippincott-Raven Press; 1996.

26. Katschnig H. Schizophrenia and quality of life. Acta Psychiatr Scand. 2000:407:33-7.

27. Diener E. Subjective well-being. Psychol Bull. 1984;95:542-75.

28. Awad AG, Voruganti LN, Heslegrave RJ. Measuring quality of life in patients with schizophrenia. PharmacoEconomics. 1997;11(1):32-47.

29. Kay SR, Fiszbein A, Opler LA. The positive and negative syndrome scale (PANSS) for schizophrenia. Schizophr Bull. 1987;13(2):261-76.

30. Endicott J, Spitzer RL, Fleiss JL, Cohen J. The global assessment scale: a procedure for measuring overall severity of psychiatric disturbance. Arch Gen Psychiatry. 1976;33(6):766-71.

31. Hall RC. Global assessment of functioning. A modified scale Psychosomatics. 1995:36(3):267-75.

32. Lingjaerde $\mathrm{O}$, Ahlfors UG, Bech P, Dencker SJ, Elgen K. The UKU side effect rating scale - a new comprehensive rating scale for psychotropic drugs and a cross-sectional study of side effects in neuroleptic-treated patients. Acta Psychiatr Scand. 1987;334:1-100.

33. Lambert M, Schimmelmann BG, Naber D, Schacht A, Karow A, Wagner T, Czekalla J. Prediction of remission as a combination of symptomatic and functional remission and adequate subjective well-being in 2960 patients with schizophrenia. J Clin Psychiatry. 2006;67:1690-7.

34. Lambert M, Naber D, Eich FX, Schacht M, Linden M, Schimmelmann BG. Remission of severely impaired subjective wellbeing in 727 patients with schizophrenia treated with amisulpride. Acta Psychiatr Scand. 2007;115:106-13.

35. Hofer A, Kemmler G, Eder U, Honeder M, Hummer M, Fleischhacker WW. Attitudes toward antipsychotics among outpatient clinic attendees with schizophrenia. J Clin Psychiatry. 2002 Jan;63(1):49-53.

36. Cohen J. A power primer. Psychol Bull. 1992;112(1):155-9.

37. Schennach-Wolff $R$, Seemüller F, Obermeier M, Messer T, Laux G, Pfeiffer H, Naber D, Schmidt LG, Gaebel W, Klosterkötter J, Heuser I, Maier W, Lemke MR, Rüther E, Buchkremer G, Gastpar M, Jäger M, Möller HJ, Riedel M. Response and remission of subjective well-being in patients suffering from schizophrenia spectrum disorders. Eur Psychiatry. 2011; 26(5):284-92. https://doi.org/10.1016/j.eurpsy.2009.11.005.

38. Wehmeier PM, Kluge M, Schacht A, Helsberg K, Schreiber W, Schimmelmann BG, Lambert M. Patterns of physician and patient rated quality of life during antipsychotic treatment in outpatients with schizophrenia. J Psychiatr Res. 2008;42(8):676-83.

39. Lambert M, Schimmelmann BG, Naber D, Eich FX, Schulz H, Huber CG, Karow A. Early- and delayed antipsychotic response and prediction of outcome in 528 severely impaired patients with schizophrenia treated with amisulpride. Pharmacopsychiatry. 2009;42(6):277-83. https://doi.org/10.1055/ s-0029-1234105.

40. Lambert M, Schimmelmann BG, Schacht A, Karow A, Wagner T, Wehmeier PM, Huber CG, Hundemer HP, Dittmann RW, Naber D. Long-term patterns of subjective wellbeing in schizophrenia: cluster, predictors of cluster affiliation, and their relation to recovery criteria in 2842 patients followed over 3 years. Schizophr Res. 2009;107(2-3):165-72. https://doi.org/10.1016/j. schres.2008.08.035

41. Larsen EB, Gerlach J. Subjective experience of treatment, side-effects, mental state and quality of life in schizophrenic outpatients treated with depot neuroleptics. Acta Psychiatr Scand. 1996;93(5):381-8.

42. Dan A, Kumar S, Avasthi A, Grover S. A comparative study on quality of life of patients of schizophrenia with and without depression. Psychiatry Res. 2011;189(2):185-9. https://doi.org/10.1016/j.psychres.2011.02.017.

43. Solanki RK, Singh P, Midha A, Chugh K. Schizophrenia: impact on quality of life. Indian J Psychiatry. 2008;50:181-6. https://doi.org/10.4103/0019-5545.43632.

44. Mas-Expósito L, Amador-Campos JA, Gómez-Benito J, Lalucat-Jo L. Research Group on Severe Mental Disorder. The World Health Organization quality of life scale brief version: a validation study in patients with schizophrenia. Qual Life Res. 2011;20(7):1079-89. https://doi.org/10.1007/s11136-011-9847-1.

45. Hosseini SH, Karkhaneh Yousefi M. Quality of life and GAF in schizophrenia correlation between quality of life and global functioning in schizophrenia. Iran J Psychiatry Behav Sci. 2011;5(2):120-5.

46. Campbell AH, Scalo JF, Crismon ML, Barner JC, Argo TR, Lawson KA, Miller A Attitudes Toward Medications and The Relationship To Outcomes in Patients with Schizophrenia. Clin Schizophr Relat Psychoses. 2015; https://doi.org/10.3371/CSRP.CASC.070415. 
47. Nielsen RE, Lindström E, Nielsen J, Levander S. DAI-10 is as good as DAl-30 in schizophrenia. Eur Neuropsychopharmacol. 2012;22(10):747-50. https://doi.org/10.1016/j.euroneuro.2012.02.008.

48. Wolters HA, Knegtering $\mathrm{H}$, van den Bosch RJ, Wiersma D. Effects and side effects of antipsychotic treatment in schizophrenia: pros and cons of available self-rating scales. Schizophr Res. 2009;112(1-3):114-8. https://doi. org/10.1016/j.schres.2009.03.026.

49. Mauri M, Mauri MC, Adami M, Reggiardo G, Giulio C. Efficacy and tolerability of paliperidone ER in patients with unsatisfactorily controlled schizophrenia by other antipsychotics: a flexible-dose approach. Int Clin Psychopharmacol. 2015;30(6):329-37. https://doi.org/10.1097/YIC.0000000000000092.

50. Marder SR. Subjective experiences on antipsychotic medications: synthesis and conclusions. Acta Psychiatr Scand Suppl. 2005:427:43-6.

Ready to submit your research? Choose BMC and benefit from:

- fast, convenient online submission

- thorough peer review by experienced researchers in your field

- rapid publication on acceptance

- support for research data, including large and complex data types

- gold Open Access which fosters wider collaboration and increased citations

- maximum visibility for your research: over $100 \mathrm{M}$ website views per year

At BMC, research is always in progress.

Learn more biomedcentral.com/submissions 